\title{
Comparison of Different Analytic Solutions to Axisymmetric Squeezing Fluid Flow between Two Infinite Parallel Plates with Slip Boundary Conditions
}

\author{
Hamid Khan, ${ }^{1}$ S. Islam, ${ }^{2}$ Javed $^{A l i},{ }^{3}$ and Inayat Ali Shah ${ }^{1}$ \\ ${ }^{1}$ Islamia College Peshawar (Chartered University), Khyber Pakhtunkhawa, Peshawar 25120, Pakistan \\ ${ }^{2}$ Department of Mathematics, CIIT, H-8, Islamabad 44000, Pakistan \\ ${ }^{3}$ CECOS University of IT and Emerging Sciences, Peshawar 25100, Pakistan
}

Correspondence should be addressed to S. Islam, saeed@comsats.edu.pk

Received 3 August 2011; Revised 6 October 2011; Accepted 6 October 2011

Academic Editor: Ahmet Yıldırım

Copyright (c) 2012 Hamid Khan et al. This is an open access article distributed under the Creative Commons Attribution License, which permits unrestricted use, distribution, and reproduction in any medium, provided the original work is properly cited.

We investigate squeezing flow between two large parallel plates by transforming the basic governing equations of the first grade fluid to an ordinary nonlinear differential equation using the stream functions $u_{r}(r, z, t)=(1 / r)(\partial \psi / \partial z)$ and $u_{z}(r, z, t)=-(1 / r)(\partial \psi / \partial r)$ and a transformation $\psi(r, z)=r^{2} F(z)$. The velocity profiles are investigated through various analytical techniques like Adomian decomposition method, new iterative method, homotopy perturbation, optimal homotopy asymptotic method, and differential transform method.

\section{Introduction}

The study of squeezing flows has widespread applications in chemical engineering, industrial engineering, mechanical engineering, biomechanics, and food industry. Valves and diarthrodial joints are also the examples for squeeze flows relevant in biology and bioengineering. The first application to squeeze flow problem was made by Stefan in 1874 [1]. The motion of a thin film of lubricant, squeezed flow between two stationary parallel plane surfaces were reported by Tichy and Winner [2] and Wang and Watson [3]. The theoretical and experimental studies of squeezing flows have been conducted by many researchers [411]. The mathematical studies of these flows are concerned primarily with the nonlinear partial differential equations which arise from the Navier-Stokes equations. These equations have no general solutions, and only a few exact solutions have been attained by confining some physical aspects of the original problem [12]. To solve these nonlinear differential 
equations, different perturbation and analytical techniques have been extensively used in fluid mechanics and engineering [13].

In the literature only a few papers deal with the comparison of different analytical methods. In this paper we study the squeezed flow between two large parallel plates with slip boundary conditions. The velocity profile is obtained using various analytical techniques like Adomian decomposition method (ADM), new iterative method (NIM), homotopy perturbation (HPM), optimal homotopy asymptotic method (OHAM), and differential transform method (DTM) [14-23]. The residual of each technique is computed and a comparison is made to assess the efficiency of the above techniques. We select DTM for analyzing the velocity profile under different flow parameters.

Squeezing flows are produced by vertical movements of boundaries or by applying external normal forces. Commonly two types of boundary conditions are employed. For a viscous fluid at a solid wall, it is generally accepted that the fluid velocity matches the velocity of the solid boundary, and it is known as no slip boundary condition. While the noslip condition is experimentally proven to be accurate for a number of macroscopic flows. Navier [24] proposed a general boundary condition that incorporates the possibility of fluid slip at a solid boundary. He assumed that the velocity $u_{x}$ at a solid surface is proportional to the shear rate at the surface, that is, $u_{x}=\beta \partial u_{x} / \partial y$, where $\beta$ is the slip length or slip coefficient. If $\beta=0$, the generally assumed no-slip boundary condition is obtained, and if $\beta$ a finite constant, fluid is slip occurs at the wall. Its effect depends upon the length scale of the flow [25-28].

\section{Basic Equation}

We consider a steady axisymmetric flow where the velocity vector, $\tilde{u}$ is represented by

$$
\tilde{u}=\left[u_{r}(r, z), 0, u_{z}(r, z)\right] \text {. }
$$

In the absence of body forces, the Navier-Stokes equations are obtained for the first grade fluid by using equations of continuity and momentum,

$$
\begin{gathered}
\tilde{\nabla} \cdot \tilde{u}=0, \\
-\rho(\tilde{u} \times \tilde{w})+\tilde{\nabla}\left(\frac{\rho}{2}|\tilde{u}|^{2}+p\right)=-\eta \tilde{\nabla} \times \widetilde{w},
\end{gathered}
$$

where $\rho$ is the constant density, $p$ is the pressure, $\eta$ is the viscosity, and $\widetilde{w}=\widetilde{\nabla} \times \tilde{u}$ is the vorticity vector.

Substituting (2.1) into (2.2), we get the following Continuity equation:

$$
\frac{\partial u_{r}}{\partial r}+\frac{u_{r}}{r}+\frac{\partial u_{z}}{\partial z}=0
$$

r-component of N.S equation:

$$
\frac{\partial}{\partial r}\left(\frac{\rho}{2}\left(u_{r}^{2}+u_{z}^{2}\right)+p\right)-\rho u_{z} \Omega(r, z)=-\eta \frac{\partial \Omega(r, z)}{\partial z} .
$$


$z$-component of N.S equation:

$$
\frac{\partial}{\partial z}\left(\frac{\rho}{2}\left(u_{r}^{2}+u_{z}^{2}\right)+p\right)-\rho u_{z} \Omega(r, z)=\eta \frac{1}{r} \frac{\partial(r \Omega(r, z))}{\partial r}
$$

where $\Omega(r, z)=\partial u_{r} / \partial z-\partial u_{z} / \partial r$ is the vorticity function.

Now we define a function which is known as generalized pressure,

$$
\widehat{p}=\frac{\rho}{2}\left(u_{r}^{2}+u_{z}^{2}\right)+p
$$

Using (2.6), (2.4) and (2.5) take the following form:

$$
\begin{gathered}
\frac{\partial}{\partial r} \widehat{p}-\rho u_{z} \Omega(r, z)=-\eta \frac{\partial \Omega(r, z)}{\partial z}, \\
\frac{\partial}{\partial z} \hat{p}-\rho u_{z} \Omega(r, z)=\eta \frac{1}{r} \frac{\partial(r \Omega(r, z))}{\partial r} .
\end{gathered}
$$

We now introduce the stream functions, $u_{r}(r, z, t)=(1 / r)(\partial \psi / \partial z), u_{z}(r, z, t)=(-1 / r) \times$ $(\partial \psi / \partial r)$, and obtain the following results:

$$
\Omega(r, z)=-\frac{1}{r} E^{2} \psi
$$

where $E^{2}=\partial^{2} / \partial r^{2}-(1 / r)(\partial / \partial r)+\partial^{2} / \partial z^{2}$,

$$
\begin{gathered}
\frac{\partial}{\partial r} \widehat{p}-\rho \frac{1}{r^{2}} \frac{\partial \psi}{\partial r} E^{2} \psi=-\frac{\eta}{r} \frac{\partial}{\partial z} E^{2} \psi, \\
\frac{\partial}{\partial z} \widehat{p}-\rho \frac{1}{r^{2}} \frac{\partial \psi}{\partial z} E^{2} \psi=-\frac{\eta}{r} \frac{\partial}{\partial r} E^{2} \psi
\end{gathered}
$$

Eliminating $\hat{p}$, from (2.9), we obtain

$$
-\rho \frac{\partial\left(\psi, E^{2} \psi / r^{2}\right)}{\partial(r, z)}=\frac{\eta}{r} E^{4} \psi
$$

We consider viscous incompressible fluid, squeezed between two large planar and parallel plates, separated by a distance $2 d$. The plates are moving towards each other with velocity $U$. The surfaces of both plates are covered by special material with slip length (slip coefficient) $\beta$. For small values of $U$ the gape distance $2 d$ between the plates varies slowly with the time $t$, so the flow can be taken as quasisteady [29-32]. See Figures 1, 2, 3, and 4. 

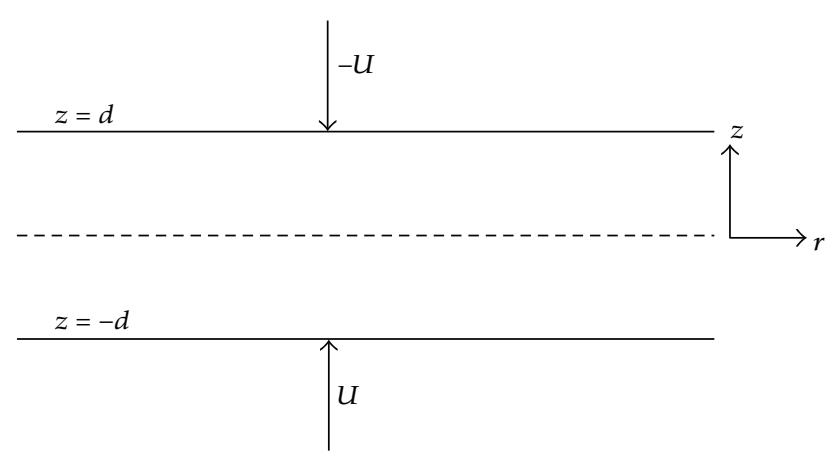

The boundary conditions are as follows:

$$
\begin{aligned}
\text { (i) At } z=d, & u_{r}=\beta \frac{\partial u_{r}}{\partial z}, & u_{z}=-U . \\
\text { (ii) At } z=0, & u_{z}=0, & \frac{\partial u_{r}}{\partial z}=0 .
\end{aligned}
$$

Now we use the transformation

$$
\psi(r, z)=r^{2} f(z)
$$

By virtue of $E^{2}=\partial^{2} / \partial r^{2}-(1 / r)(\partial / \partial r)+\partial^{2} / \partial z^{2}$ and (2.12), the compatibility equation (2.10) and the boundary conditions equation (2.11) become

$$
\begin{gathered}
F^{i v}(z)+2 \frac{\rho}{\eta} F(z) F^{\prime \prime \prime}(z)=0, \\
F(0)=0, \quad F^{\prime \prime}(0)=0, \quad F(d)=\frac{U}{2}, \quad F^{\prime}(d)=\beta F^{\prime \prime}(d) .
\end{gathered}
$$

Now by introducing dimensionless parameters

$$
F^{*}=\frac{F}{U / 2}, \quad z^{*}=\frac{z}{d}, \quad \gamma=\frac{\beta}{d}, \quad R=\frac{\rho d U}{\eta},
$$

and dropping “*” for simplicity, the boundary value problem (2.13) become,

$$
\frac{d^{4} F}{d z^{4}}+R F \frac{d^{3} F}{d z^{3}}=0
$$

with boundary conditions

$$
F(0)=0, \quad F^{\prime \prime}(0)=0, \quad F(1)=1, \quad F^{\prime}(1)=\gamma F^{\prime \prime}(1) .
$$




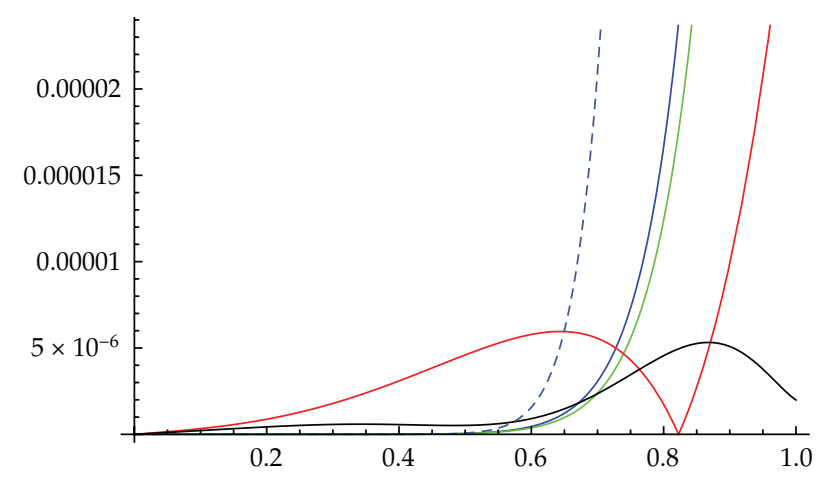

Figure 1: Plots of the residuals, blue: ADM, green: NIM, red: HPM, black: OHAM and dashed: DTM. In this figure, a comparison of the residuals is shown graphically, and it reveals that OHAM is more suitable technique for this problem. One can easily observe that the curve of the OHAM residual is approximately normally distributed while the curves of the residuals of other methods are $J$-shaped and highly skewed.

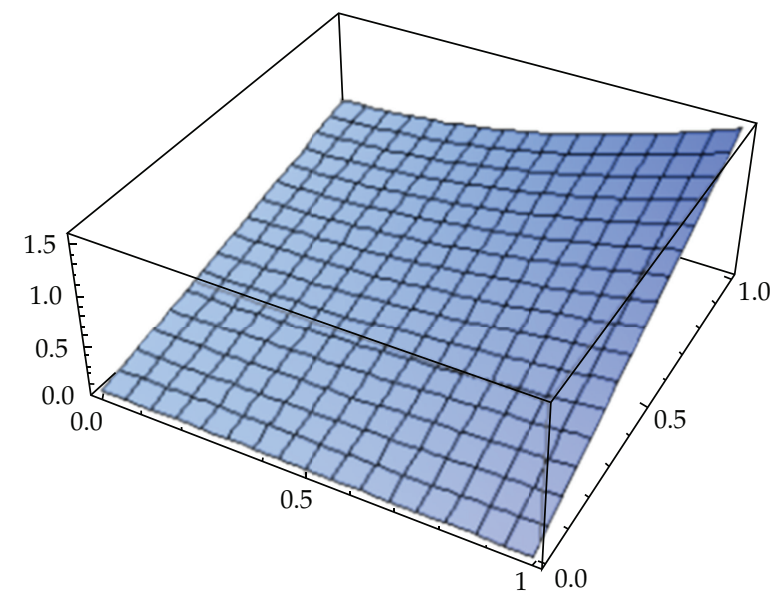

Figure 2: $z$-component $u_{z}(r, \theta, z)=(-1 / r)(\partial \psi / \partial r)$ of the velocity profile given by OHAM, when $R=1, r=$ 1 .

\section{Analytical Techniques}

In this section, we give the basic idea of various analytical techniques and evaluate the velocity profile of our problem by considering $R=1, \gamma=1$. The residuals of all the techniques are computed and the results are displayed in Table 2.

\subsection{Adomian Decomposition Method}

According to $[13,14]$, we consider the differential equation

$$
L(F(z))+M(F(z))+N(F(z))=g(z)
$$




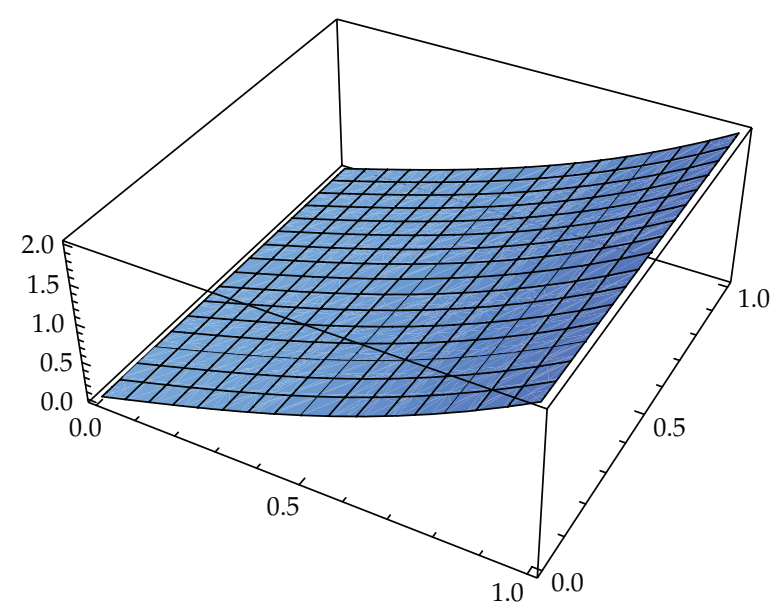

Figure 3: $r$-component $u_{r}(r, \theta, z)=(1 / r)(\partial \psi / \partial z)$ of the velocity profile given by OHAM, when $R=1$, $\gamma=1$.

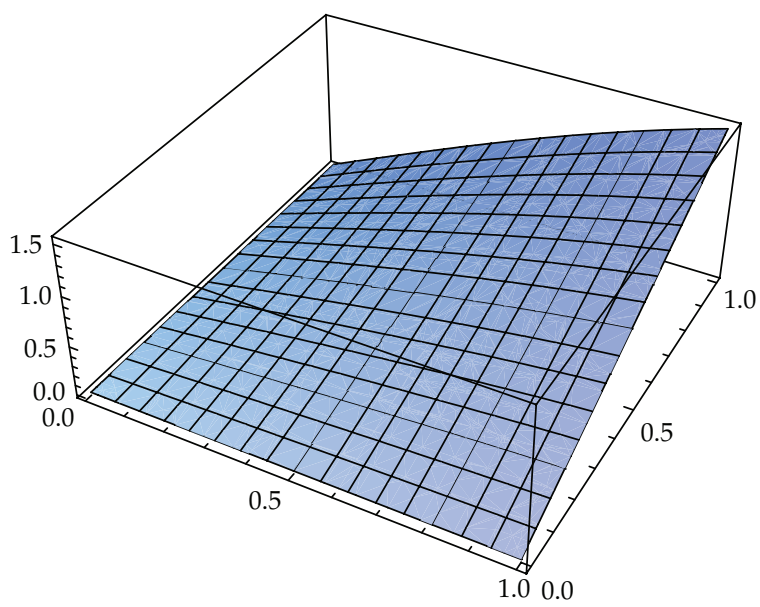

Figure 4: Vorticity function $\Omega(r, z)=\partial u_{r} / \partial z-\partial u_{z} / \partial r$ where the velocity components have been obtained by OHAM, for $R=1, \gamma=1$.

where $L$ is the operator of the highest order derivative with respect to $z, L=d^{4} / d z^{4}, R$ is the reminder of the linear term, and the nonlinear term is represented by $N(F(z))$. Operating $L^{-1}$ on both sides of (3.1) we get the following:

$$
F(z)=\alpha_{0}+\alpha_{1} z+\alpha_{2} \frac{z^{2}}{2 !}+\alpha_{3} \frac{z^{3}}{3 !}+L^{-1}(g(z))-L^{-1} M(F(z))-L^{-1} N(F(z)),
$$

where $\alpha_{i}: i=0,1,2,3$, the constants can be determined by using initial or boundary conditions. 
The unknown function $F(z)$ can be expressed by an infinite series of the form

$$
F(z)=\sum_{n=0}^{\infty} F_{n}(z)
$$

where $F_{0}(z)=\alpha_{0}+\alpha_{1} z+\alpha_{2} z^{2} / 2 !+\alpha_{3} z^{3} / 3 !+L^{-1}(g(z))$ and $F_{n+1}=-L^{-1} M\left(F_{n}(z)\right)-L^{-1}\left(A_{n}\right)$, $n=0,1,2, \ldots$. The nonlinear term $N(F(z))$ is decomposed by an infinite series of polynomial given by

$$
N(F(z))=\sum_{n=0}^{\infty} A_{n}
$$

where $A_{n}$ are the so-called Adomian polynomials that can be determined by the formula

$$
A_{n}=\frac{1}{n !} \frac{d^{n}}{d \lambda^{n}}\left[N\left(\sum_{i=0}^{n} \lambda^{i} F_{i}\right)\right]_{\lambda=0} .
$$

It has been observed that these polynomials can be constructed for a wide class of nonlinear functions.

The solution $F(z)=\sum_{n=0}^{\infty} F_{n}(z)$ is approximated by the truncated series of order $K$, that is,

$$
\tilde{F}(z)=\sum_{n=0}^{K} F_{n}(z)
$$

In our case $g(z)=0, M(F(z))=0$.

Now appling ADM on (2.15) and (2.16), for $n=0,1, \ldots, 5$.

We obtain

$$
\begin{gathered}
F_{0}(z)=A \frac{z^{3}}{6}+B z \\
F_{1}(z)=-\left(\frac{1}{120} A B z^{5}+\frac{A^{2} z^{7}}{5040}\right) \\
F_{2}(z)=\frac{A B^{2} z^{7}}{1680}+\frac{A^{2} B z^{9}}{22680}+\frac{A^{3} z^{11}}{1108800} \\
\vdots
\end{gathered}
$$

Considering the Adomian 5th-order solution,

$$
\tilde{F}(z)=F_{0}(z)+F_{1}(z)+\cdots+F_{5}(z)+O\left(z^{20}\right) .
$$


The boundary conditions at $z=1$ are used to get the following values of $A$ and $B$.

$$
A=1.74911, \quad B=0.71896 .
$$

By substituting these values our solution is

$$
\begin{aligned}
\tilde{F}(z)= & 0.718965 z+0.291518 z^{3}-0.0104795 z^{5}-0.0000688452 z^{7}+0.0000701133 z^{9} \\
& -3.49247 \times 10^{-6} z^{11}-4.73949 \times 10^{-7} z^{13}+5.95015 \times 10^{-8} z^{15} \\
& -8.90909 \times 10^{-10} z^{17}-1.65991 \times 10^{-9} z^{19}+O\left(z^{20}\right)
\end{aligned}
$$

\subsection{New Iterative Method}

The basic idea of new iterative method $[15,16]$. Consider the following nonlinear general differential equation:

$$
L(F(z))+M(F(z))+N(F(z))=g(z)
$$

where $L$ is the operator of the highest order derivative with respect to $z, L=d^{4} / d z^{4}, M$ is the reminder of the linear term, and the nonlinear term is represented by $N(F(z))$. Operating $L^{-1}$ on both sides of (3.11) we get,

$$
F(z)=\alpha_{0}+\alpha_{1} z+\alpha_{2} \frac{z^{2}}{2 !}+\alpha_{3} \frac{z^{3}}{3 !}+L^{-1}(g(z))-L^{-1} M(F(z))-L^{-1} N(F(z))
$$

where $\alpha_{i}: i=0,1,2,3$, are constants to be determined by using initial or boundary conditions.

The unknown function $F(z)$ can be expressed by an infinite series of the form

$$
F(z)=\sum_{n=0}^{\infty} F_{n}(z)
$$

where $F_{0}(z)=\alpha_{0}+\alpha_{1} z+\alpha_{2} z^{2} / 2 !+\alpha_{3} z^{3} / 3 !+L^{-1} g(z)$ and $F_{n+1}=-L^{-1} M\left(F_{n}(z)\right)-L^{-1}\left(G_{n}\right)$, $n=0,1,2, \ldots$ The nonlinear term $N(F(z))$ is decomposed by an infinite series of polynomials given by

$$
N(F(z))=\sum_{n=0}^{\infty} G_{n}
$$

where $G_{n}=N\left(\sum_{k=0}^{n} F_{k}\right)-N\left(\sum_{k=0}^{n-1} F_{k}\right)$ and $G_{0}=N\left(F_{0}(z)\right)$. 
The solution $F(z)=\sum_{n=0}^{\infty} F_{n}(z)$, is approximated by the truncated series of order $K$, that is,

$$
\widetilde{F}(z)=\sum_{n=0}^{K} F_{n}(z) .
$$

In our case $g(z)=0$ and $M(F(z))=0$.

Now using NIM on (2.15) and (2.16), for $n=0,1, \ldots, 5$.

We obtain the following:

$$
\begin{gathered}
F_{0}(z)=A \frac{z^{3}}{6}+B z \\
F_{1}(z)=-\left(\frac{1}{120} A B z^{5}+\frac{A^{2} z^{7}}{5040}\right) \\
F_{2}(z)=-\frac{A B^{2} z^{7}}{1680}+\frac{A^{2} B z^{9}}{22680}+\frac{A^{3} z^{11}}{1108800}-\frac{A^{2} B^{2} z^{11}}{1900800}-\frac{A^{3} B z^{13}}{38438400}-\frac{A^{4} z^{15}}{3962649600}
\end{gathered}
$$

Considering the NIM 5th-order solution,

$$
\tilde{F}(z)=F_{0}(z)+F_{1}(z)+F_{3}(z)+F_{4}(z)+F_{5}(z)+O\left(z^{20}\right)
$$

we have the following:

$$
\begin{aligned}
\tilde{F}(z)= & B z+\frac{A z^{3}}{6}-\frac{1}{120} A B z^{5}+\left(-\frac{A^{2}}{5040}+\frac{A B^{2}}{1680}\right) z^{7}+\left(\frac{A^{2} B}{22680}-\frac{A B^{3}}{24192}\right) z^{9} \\
& +\left(\frac{A^{3}}{1108800}-\frac{241 A^{2} B^{2}}{39916800}+\frac{A B^{4}}{380160}\right) z^{11}+\left(-\frac{71 A^{3} B}{239500800}+\frac{131 A^{2} B^{3}}{207567360}-\frac{A B^{5}}{6589440}\right) z^{13} \\
& +\left(-\frac{1051 A^{4}}{217945728000}+\frac{1357 A^{3} B^{2}}{25147584000}-\frac{4759 A^{2} B^{4}}{87178291200}\right) z^{15} \\
& +\left(\frac{2243 A^{4} B}{1140023808000}-\frac{359 A^{3} B^{3}}{50523782400}+\frac{179 A^{2} B^{5}}{130288435200}\right) z^{17} \\
& +\left(\frac{21919 A^{5}}{844757641728000}-\frac{71999 A^{4} B^{2}}{167094918144000}+\frac{104977 A^{3} B^{4}}{300358272614400}-\frac{31 A^{2} B^{6}}{649813570560}\right) z^{19} \\
& +O\left(z^{20}\right) .
\end{aligned}
$$

Using the boundary conditions at $z=1$, we get the following value of $A$ and $B$

$$
A=1.74911, \quad B=0.71897 .
$$


The approximate solution is as follows:

$$
\begin{aligned}
\tilde{F}(z)= & 0.718965 z+0.291518 z^{3}-0.0104795 z^{5}-0.0000688448 z^{7}+0.0000701132 z^{9} \\
& -3.49247 \times 10^{-6} z^{11}-4.73948 \times 10^{-7} z^{13}+5.95015 \times 10^{-8} z^{15} \\
& -8.34647 \times 10^{-11} z^{17}-1.18034 \times 10^{-9} z^{19}+O\left(z^{20}\right) .
\end{aligned}
$$

\subsection{HPM}

To illustrate the basic idea of homotopy perturbation method [17-21], we consider the following nonlinear differential equation:

$$
A(F)=f(r)
$$

$A$ is a general differential operator, the operator $A$ can usually be divided into two parts $L$ and $N$, where $L$ is linear, and $N$ is nonlinear:

$$
A=L+N
$$

so

$$
L(F)+N(F)-f(r)=0, \quad r \in \Omega .
$$

$f(r)$ is a known analytic function.

With the boundary condition $B(F, \partial F / \partial n)=0, r \in \Gamma$.

$B$ is a boundary operator, and $\Gamma$ is the boundary of the domain $\Omega$.

Now we construct the following homotopy:

$$
H(v, p)=(1-p)\left[L(v)-L\left(F_{0}\right)\right]+p[A v-f(r)]
$$

where $p \in[0,1]$ is an embedding parameter and $F_{0}$ is the first approximation that satisfied the boundary condition. To get an approximate solution, we expand $F(r, p)$ in Taylor's series about $p$ in the following manner:

$$
F(r)=v_{0}(r)+\sum_{m=1}^{\infty} v_{m}(r) p^{m}
$$

Plugging (3.25) into (3.24) and then equating the coefficient of like powers of $p$, we get the following problems which are directly integrable.

Zeroth-order problem:

$$
\begin{gathered}
F_{0}^{(i v)}(z)=0, \\
F_{0}(0)=0, \quad F_{0}^{\prime \prime}(0)=0, \quad F_{0}(1)=1, \quad F_{0}^{\prime}(1)=F_{0}^{\prime \prime}(1) .
\end{gathered}
$$


First-order problem:

$$
\begin{gathered}
F_{1}^{i v}(z)=-F_{0}(z) F_{0}^{\prime \prime \prime}(z), \\
F_{1}(0)=0, \quad F_{1}^{\prime \prime}(0)=0, \quad F_{1}(1)=0, \quad F_{1}^{\prime}(1)=F_{1}^{\prime \prime}(1) .
\end{gathered}
$$

Second-order problem:

$$
\begin{gathered}
F_{2}^{i v}(z)=-F_{1}(z) F_{0}^{\prime \prime \prime}(z)-F_{0}(z) F_{1}^{\prime \prime \prime}(z), \\
F_{2}(0)=0, F_{2}^{\prime \prime}(0)=0, F_{2}(1)=0, F_{2}^{\prime}(1)=F_{2}^{\prime \prime}(1)
\end{gathered}
$$

We consider the following 5th-order solution,

$$
\begin{aligned}
\tilde{F}(z)= & F_{0}(z)+F_{1}(z)+F_{3}(z)+F_{4}(z)+F_{5}(z)+O\left(z^{20}\right) \\
\tilde{F}(z)= & \frac{2379640217780939164049 z}{3309814671645081600000}+\frac{723651501050808628889 z^{3}}{2482361003733811200000}-\frac{43087465806546383 z^{5}}{4111571020677120000} \\
& -\frac{1652256496336207 z^{7}}{23984164287283200000}+\frac{121738793951 z^{9}}{1735816642560000}-\frac{31017787 z^{11}}{8879270400000} \\
& -\frac{430067207 z^{13}}{909237288960000}+\frac{74925133 z^{15}}{1223973273600000}+\frac{30859 z^{17}}{15429481267200} \\
& -\frac{16191589 z^{19}}{18688959184896000}+O\left(z^{20}\right)
\end{aligned}
$$

\subsection{OHAM}

According to [22-24], we consider the following differential equation:

$$
A(F(z))=f(r), \quad r \in \Omega,
$$

with the boundary conditions

$$
B\left(F, \frac{\partial F(z)}{\partial n}\right)=0, \quad r \in \Gamma
$$

where $A$ is a differential operator, $B$ is a boundary operator, and $f(r)$ is a known function of $r: r \in \Omega$. The operator $A$ can be written as $A=L+N$, where $L$ is linear and $N$ is a nonlinear operator. In OHAM we first construct a homotopy equation,

$$
(1-p) L(F(r, p))-h(p)[L(F(r, p))+N(F(r, p))-f(r)]=0
$$


where $p \in[0,1]$ is an embedding parameter, $h(p)$ is a nonzero auxiliary function for $p \neq 0$ and $h(0)=0$

$$
L(F(z))=0, \quad \text { for } p=0 \quad A(F(z))=f(r), \quad \text { for } p=1 .
$$

The solution $F(r, 0)=v_{0}(r)$ of $L(F(z))=0$ traces the solution curve $v(r)$ continuously as $p$ approaches to 1 , where $v_{0}$ is the solution of the zeroth-order problem that will come in the next few lines. We next choose the auxiliary function $h(p)$ in the following form:

$$
h(p)=\sum_{i=1}^{m} p^{i} C_{i}
$$

where $C_{1}, C_{2}, \ldots$ are the convergence controlling constants which are to be determined. To get an approximate solution, we expand $F(r, p)$ in Taylor's series about $p$ in the following manner:

$$
F\left(r, p, C_{i}\right)=v_{0}(r)+\sum_{m=1}^{\infty}\left(r, C_{1}, C_{1}, \ldots, C_{m}\right) p^{m}
$$

Now after substituting the auxiliary function $h(p)$ and $F\left(r, p, C_{i}\right)$ in homotopy equation we compare the coefficient of like powers of $p$, to obtain the following linear equations.

Zeroth-order problem:

$$
L v_{0}=0, \quad B\left(v_{0}, \frac{\partial v_{0}}{\partial n}\right)=0 .
$$

First-order problem:

$$
L v_{1}-\left(1+C_{1} v_{0}\right) L+C_{1} f(r)=C_{1} N_{0} v_{0}, \quad B\left(v_{1}, \frac{\partial v_{1}}{\partial n}\right)=0
$$

Second-order problem:

$$
L v_{2}-\left(1+C_{1}\right) L v_{1}-C_{2} L v_{0}-C_{2} f(r)=C_{2} N_{0} v_{0}+C_{1} N_{1} v_{1}, \quad B\left(v_{2}, \frac{\partial v_{2}}{\partial n}\right)=0,
$$

and so on.

If the series is convergent at $p=1$ for suitable auxiliary constants $C_{1}, C_{2}, \ldots$, then

$$
v(r)=F\left(r, C_{i}\right)=v_{0}(r)+\sum_{m=1}^{\infty} v_{m}\left(r, C_{1}, C_{1}, \ldots, C_{m}\right)
$$


The result of the mth-order approximations are given by

$$
\breve{v}(r)=v_{0}(r)+\sum_{i=1}^{m} v_{i}\left(r, C_{1}, C_{1}, \ldots, C_{i}\right)
$$

Residual of the solution is

$$
R\left(r, C_{1}, C_{2}, \ldots, C_{m}\right)=A \breve{v}-f(r) .
$$

If $R=0, \breve{v}$ will be the exact solution, but it does not happen specially in nonlinear problems. To find the optimal values of $C_{i}$, many methods can be applied. We follow the method of least squares. According to the method of least squares, we first construct the functional

$$
J\left(r, C_{1}, C_{2}, \ldots, C_{m}\right)=\int_{a}^{b} R^{2} d r
$$

and then minimizing it, we have

$$
\frac{\partial J}{\partial C_{1}}=\frac{\partial J}{\partial C_{2}}=\cdots=\frac{\partial J}{\partial C_{m}}=0
$$

where $a$ and $b$ are in the domain of the problem. With these constants known, the approximate solution (of order $m$ ) is well determined.

Now applying OHAM to (2.15) and (2.16), we obtain the following problems which are directly integrable.

Zeroth-order problem:

$$
\begin{gathered}
F_{0}^{(i v)}(z)=0, \\
F_{0}(0)=0, \quad F_{0}^{\prime \prime}(0)=0, \quad F_{0}(1)=1, \quad F_{0}^{\prime}(1)=F_{0}^{\prime \prime}(1) .
\end{gathered}
$$

First-order problem:

$$
\begin{gathered}
F_{1}^{i v}(z)=C_{1} F_{0}(z) F_{0}^{\prime \prime \prime}(z)+F_{0}^{i v}(z)+C_{1} F_{0}^{i v}(z), \\
F_{1}(0)=0, \quad F_{1}^{\prime \prime}(0)=0, \quad F_{1}(1)=0, \quad F_{1}^{\prime}(1)=F^{\prime \prime}{ }_{1}(1) .
\end{gathered}
$$

Second-order problem:

$$
\begin{gathered}
F_{2}^{i v}(z)=C_{1} F_{1}(z) F_{0}^{\prime \prime \prime}(z)+C_{1} F_{0}(z) F_{1}^{\prime \prime \prime}(z)+F_{1}^{i v}(z)+C_{1} F_{1}^{i v}(z), \\
F_{2}(0)=0, \quad F_{2}^{\prime \prime}(0)=0, \quad F_{2}(1)=0, \quad F_{2}^{\prime}(1)=F_{2}^{\prime \prime}(1) \\
\vdots
\end{gathered}
$$


Considering the OHAM 5th-order solution and using the method of least squares, we obtain $C_{1}=-0.93281$. Hence the solution is

$$
\begin{aligned}
\tilde{F}(z)= & 0.718965 z+0.291517 z^{3}-0.0104795 z^{5}-0.0000688407 z^{7}+0.0000700828 z^{9} \\
& -3.4536 \times 10^{-6} z^{11}-4.87073 \times 10^{-7} z^{13}+5.80616 \times 10^{-8} z^{15} \\
& +3.31269 \times 10^{-9} z^{17}-5.61766 \times 10^{-10} z^{19}+O\left(z^{20}\right) .
\end{aligned}
$$

\subsection{NDSolve}

NDSolve is a mathematica code, utilized for solution of ordinary and partial differential equations. This code is also used for differential-algebraic equations and system of ordinary differential equation. NDSolve gives solution on discrete points rather than for the function $F$ itself. List interpolation is used for the construction of approximating polynomial.

Apply NDSolve to (2.15) and (2.16), the following approximate solution is obtained:

$$
\begin{aligned}
F(z)= & 0.718965 z-2.5833 \times 10^{-6} z^{2}+0.291529 z^{3}-0.000025575 z^{4}-0.0104737 z^{5} \\
& +0.0000970056 z^{6}-0.000294814 z^{7}+0.000231011 z^{8}-0.0000378459 z^{9} \\
& +0.0000122062 z^{10}
\end{aligned}
$$

\subsection{DTM}

According to $[33,34]$, the basic idea of differential transforms method (DTM) starts from the following definition.

If $F(z)$ is a given function, its differential transform is defined as follows:

$$
\bar{F}(r)=\left.\frac{1}{r !} \frac{d^{r} F(z)}{d z^{r}}\right|_{z=0} .
$$

The inverse transform of $\bar{F}(r)$ is defined by

$$
F(z)=\sum_{r=0}^{\infty} z^{r} \bar{F}(r)
$$

In actual application, the function $F(z)$ is expressed by a finite series

$$
F(z)=\sum_{r=0}^{N} z^{r} \bar{F}(r) .
$$

Equation (3.51) implies that $F(z)=\sum_{r=N+1}^{\infty} z^{r} \bar{F}(r)$. is negligibly small.

The fundamental operations of the DTM are given in Table 1. 
Table 1

\begin{tabular}{lc}
\hline Function & Transformed function \\
\hline$K(z)=\alpha K_{1}(z) \pm \beta K_{2}(z)$ & $K(r)=\alpha \bar{K}_{1}(r) \pm \beta \bar{K}_{2}(r)$ \\
$K(z)=F^{(m)}(z)$ & $K(r)=\frac{(m+r) !}{r !} F(m+r)$ \\
$K(z)=K_{1}(z) K_{2}(z)$ & $\bar{K}(r)=\sum_{n=0}^{r} \bar{K}_{1}(n) \bar{K}_{2}(r-n)$ \\
$K(z)=z^{m}$ & $\bar{K}(r)=\delta(r-m), \delta(p)=\left\{\begin{array}{cc}1, & p=0 \\
0, & p \neq 0\end{array}\right.$ \\
$K(z)=K_{1}(z) K_{2}(z) \cdots K_{m}(z)$ & $G(r)=\sum_{l_{m-1=0}}^{r} \sum_{l_{m-2=0}}^{l_{m-1} \cdots \sum_{l_{2=0}}^{l_{3}} \sum_{l_{1=0}}^{l_{2}}\left\{\bar{K}_{1}\left(l_{1}\right) \bar{K}_{2}\left(l_{2}-l_{1}\right) \cdots \bar{K}_{m}\left(r-l_{m-1}\right)\right\}}$ \\
\hline
\end{tabular}

\subsubsection{Analysis of the Method}

Consider a fourth-order boundary value problem

$$
F^{(4)}(z)=G(z, F), \quad 0<z<H,
$$

with the boundary conditions:

$$
F(0)=\alpha_{0}, \quad F(H)=\alpha_{1}, \quad F^{(1)}(H)=\alpha_{2}, \quad F^{(2)}(0)=\alpha_{3},
$$

where $\alpha_{i}: i=0,1,2,3$ are given values.

The differential transform of (3.52) is as follows:

$$
\bar{F}(r+4)=\frac{\bar{G}(r)}{\prod_{i=1}^{4}(r+i)},
$$

where $\bar{G}(r)$ is the differential transform of $G(z, F)$.

The transformed boundary conditions (3.53) are given by

$$
\bar{F}(0)=\alpha_{0}, \quad \sum_{r=0}^{N} H^{r} \bar{F}(r)=\alpha_{1}, \quad \sum_{r=0}^{N} r H^{r} \bar{F}(r)=\alpha_{2}, \quad \bar{F}(2)=\frac{\alpha_{3}}{2} .
$$

Using (3.54) and (3.55) values of $\bar{F}(i): i=4,5, \ldots$ are obtained which give the following series solution up to $O\left(z^{N+1}\right)$,

$$
F(z)=\sum_{r=0}^{N} z^{r} \bar{F}(r)+O\left(z^{N+1}\right),
$$


Table 2: Comparison of residuals. In this table residuals of all the techniques at the different domain points are displayed. We observe that OHAM provides more stable solution.

\begin{tabular}{lllllll}
\hline $\mathrm{z}$ & ADM & NIM & HPM & OHAM & NDSolve & DTM \\
\hline 0 & 0 & 0 & 0 & 0 & $-6.14 E-4$ & 0 \\
0.1 & $-6.66 E-16$ & $-6.94 E-16$ & $-3.28 E-7$ & $2.18 E-7$ & $-3.47 E-4$ & $2.22 E-16$ \\
0.2 & $-1.40 E-12$ & $-1.36 E-12$ & $-8.73 E-7$ & $4.31 E-7$ & $-7.35 E-5$ & $-1.26 E-14$ \\
0.3 & $-1.35 E-10$ & $-1.27 E-10$ & $-1.79 E-6$ & $5.72 E-7$ & $1.70 E-5$ & $-1.26 E-11$ \\
0.4 & $-3.66 E-9$ & $-3.30 E-9$ & $-3.09 E-6$ & $5.64 E-7$ & $-5.16 E-7$ & $-1.65 E-9$ \\
0.5 & $-5.05 E-8$ & $-4.34 E-8$ & $-4.60 E-6$ & $5.17 E-7$ & $-1.14 E-5$ & $-7.20 E-8$ \\
0.6 & $-4.55 E-7$ & $-3.72 E-7$ & $-5.78 E-6$ & $9.07 E-7$ & $1.16 E-5$ & $-1.56 E-6$ \\
0.7 & $-3.06 E-6$ & $-2.38 E-6$ & $-5.56 E-6$ & $2.35 E-6$ & $2.11 E-6$ & $-2.09 E-5$ \\
0.8 & $-1.66 E-5$ & $-1.24 E-5$ & $-1.74 E-6$ & $4.55 E-6$ & $-5.40 E-5$ & $-1.97 E-4$ \\
0.9 & $-7.68 E-5$ & $-5.48 E-5$ & $9.89 E-6$ & $5.08 E-6$ & $2.23 E-4$ & $-1.41 E-3$ \\
1.0 & $-3.11 E-4$ & $-2.13 E-4$ & $3.60 E-5$ & $1.99 E-6$ & $2.20 E-3$ & $-8.15 E-3$ \\
\hline
\end{tabular}

applying DTM on (2.15) and (2.16), the transformed boundary conditions and differential are as follows:

$$
\begin{gathered}
\bar{F}(0)=0, \quad \bar{F}(1)=a, \quad \bar{F}(2)=0, \quad \bar{F}(3)=b, \\
\bar{F}(r+4)=\frac{r !}{(r+4)}\left\{-R \sum_{k=0}^{r}(k+1)(k+2)(k+3) \bar{F}(k+3) \bar{F}(r-k)\right\} .
\end{gathered}
$$

Using (3.57), we obtain the following values of $\bar{F}(i): i=1,2,3 \ldots, 19$.

For these values, the unknowns $a$ and $b$ are determined by the following system:

$$
\sum_{r=0}^{19} \tilde{F}(r)=1, \quad \sum_{r=0}^{19} r \widetilde{F}(r)=\sum_{r=0}^{19} r(r-1) \widetilde{F}(r)
$$

We find, $a=1.53266$ and $b=-0.570353$.

Now using the inverse differential transform, the following approximate solution of $O\left(z^{20}\right)$ is obtained:

$$
\begin{aligned}
\widetilde{F}(z)= & 1.53266 z-0.570353 z^{3}+0.043708 z^{5}-0.00710856 z^{7}+0.00130068 z^{9} \\
& -0.000251907 z^{11}+0.0000492109 z^{13}-9.6216 \times 10^{-6} z^{15} \\
& +1.86893 \times 10^{-6} z^{17}-3.60367 \times 10^{-7} z^{19}+O\left(z^{20}\right) .
\end{aligned}
$$

\section{Conclusion}

In this paper we have used Adomian's decomposition method, new iterative method, homotopy perturbation method, optimal homotopy asymptotic method, and differential 
transform method to an axisymmetric squeezing flow problem. Though all the methods are based on Taylor's series expansion, they produce different results because each one has its own environment. Homotopy methods combine homotopy from topology and perturbation method. It has been observed that homotopy perturbation method may suffer convergence in many problems while OHAM controls the convergence region by employing the auxiliary function. Differential transform method, Adomian decomposition method, and new iterative method are the straightforward application of Taylor's series, and they suffer from divergence in general and particularly in initial value problems. Besides all these facts, ADM, HPM, and DTM can lead easily to closed-form solutions of many problems.

\section{References}

[1] J. Stefan, Versuche über die Scheinbare Adhäsion, Sitzungsberichte der Kaiserlichen Akademie der Wissenschaften, Mathematisch Naturwissenschaftliche Classe, vol. 69, Abteilung Wien, 1874.

[2] J. Tichy and W. O. Winner, "Inertial considerations in parallel circular squeeze film bearings," Transactions of the ASME: Journal of Lubrication Technology, vol. 92, pp. 588-592, 1970.

[3] C. Y. Wang and L. T. Watson, "Squeezing of a viscous fluid between elliptic plates," Applied Scientific Research, vol. 35, no. 2-3, pp. 195-207, 1979.

[4] R. J. Grimm, "Squeezing flows of Newtonian liquid films an analysis including fluid inertia," Applied Scientific Research, vol. 32, no. 2, pp. 146-149, 1976.

[5] W. A. Wolfe, "Squeeze film pressures," Applied Scientific Research, vol. 14, pp. 77-90, 1964-1965.

[6] D. C. Kuzma, "Fluid inertia effects in squeeze films," Applied Scientific Research, vol. 18, no. 1, pp. 15-20, 1968.

[7] S. Ishizawa, "Squeezing flows of Newtonian liquid films an analysis including fluid inertia," Applied Scientific Research, vol. 32, no. 2, pp. 149-166, 1976.

[8] R. Usha and R. Sridharan, "Arbitrary squeezing of a viscous fluid between elliptic plates," Fluid Dynamics Research, vol. 18, no. 1, pp. 35-51, 1996.

[9] H. M. Laun, M. Rady, and O. Hassager, "Analytical solutions for squeeze flow with partial wall slip," Journal of Non-Newtonian Fluid Mechanics, vol. 81, no. 1-2, pp. 1-15, 1999.

[10] M. H. Hamdan and R. M. Barron, "Squeeze flow of dusty fluids," Applied Scientific Research, vol. 49, no. 4, pp. 345-354, 1992.

[11] P. T. Nhan, "Squeezing flow of a viscoelastic solid," Journal of Non-Newtonian Fluid Mechanics, vol. 95, no. 2-3, pp. 343-362, 2000.

[12] M. Kompani and D. C. Venerus, "Equibiaxial extensional flow of polymer melts via lubricated squeezing flow. I. Experimental analysis," Rheologica Acta, vol. 39, no. 5, pp. 444-451, 2000.

[13] L. Debnath and A. K. Ghosh, "On unsteady hydromagnetic flows of a dusty fluid between two oscillating plates," Applied Scientific Research, vol. 45, no. 4, pp. 353-365, 1988.

[14] G. Adomian, "A review of the decomposition method and some recent results for nonlinear equations," Computers \& Mathematics with Applications, vol. 21, no. 5, pp. 101-127, 1991.

[15] V. Daftardar-Gejji and H. Jafari, "An iterative method for solving nonlinear functional equations," Journal of Mathematical Analysis and Applications, vol. 316, no. 2, pp. 753-763, 2006.

[16] S. Bhalekar and V. Daftardar-Gejji, "New iterative method: application to partial differential equations," Applied Mathematics and Computation, vol. 203, no. 2, pp. 778-783, 2008.

[17] J.-H. He, "Homotopy perturbation technique," Computer Methods in Applied Mechanics and Engineering, vol. 178, no. 3-4, pp. 257-262, 1999.

[18] J.-H. He, "Homotopy perturbation method for solving boundary value problems," Physics Letters. A, vol. 350, no. 1-2, pp. 87-88, 2006.

[19] M. A. Noor and S. T. Mohyud-Din, "Homotopy method for solving eighth order boundary value problems," Journal of Mathematical Analysis and Approximation Theory, vol. 1, no. 2, pp. 161-169, 2006.

[20] M. A. Noor and S. T. Mohyud-Din, "Homotopy method for solving eighth order boundary value problems," Journal of Mathematical Analysis and Approximation Theory, vol. 1, no. 2, pp. 1-15, 2006.

[21] M. A. Noor and S. T. Mohyud-Din, "Homotopy perturbation method for solving Thomas-Fermi equation using Pade approximants," International Journal of Nonlinear Science, vol. 8, no. 1, pp. 27-31, 2009. 
[22] V. Marinca and N. Herişanu, "An optimal homotopy asymptotic method for solving nonlinear equations arising in heat transfer," International Communications in Heat and Mass Transfer, vol. 35, no. 6 , pp. 710-715, 2008.

[23] V. Marinca, N. Herişanu, C. Bota, and B. Marinca, "An optimal homotopy asymptotic method applied to the steady flow of a fourth-grade fluid past a porous plate," Applied Mathematics Letters, vol. 22, no. 2, pp. 245-251, 2009.

[24] C. L. M. H. Navier, Memoirs de l'Academie Royale des Sciences de l'Institut de France, vol. 1, Royale des Sciences de l'Institut de France, 1823.

[25] E. Lauga, M. P. Brenner, and H. A. Stone, "Microfluidics: the no-slipboundary condition," in Handbook of Experimental Fluid Dynamics, C. Tropea, A. Yarin, and J. F. Foss, Eds., pp. 1219-1240, Springer, New York, NY, USA, 2007.

[26] H. M. Laun, M. Rady, and O. Hassager, "Analytical solutions for squeeze flow with partial wall slip," Journal of Non-Newtonian Fluid Mechanics, vol. 81, no. 1-2, pp. 1-15, 1999.

[27] C. Neto, D. R. Evans, E. Bonaccurso, H. J. Butt, and V. S. J. Craig, “Boundary slip in Newtonian liquids: a review of experimental studies," Reports on Progress in Physics, vol. 68, no. 12, pp. 2859-2897, 2005.

[28] Y. Zhu and S. Granick, "Limits of the hydrodynamic no-slip boundary condition," Physical Review Letters, vol. 87, article 096105, 2001.

[29] T. C. Papanastasiou, G. C. Georgiou, and A. N. Alexandrou, Viscous Fluid Flow, CRC Press, Boca Raton, Fla, USA, 2000

[30] M. Siddiqui, M. Ahmed, and Q. K. Ghori, "Application of homotopy perturbation method to squeezing flow of a newtonian fluid," International Journal of Nonlinear Sciences and Numerical Simulation, vol. 8, no. 2, pp. 179-184, 2007.

[31] X. J. Ran, Q. Y. Zhu, and Y. Li, “An explicit series solution of the squeezing flow between two parallel plates," Communications in Nonlinear Science and Numerical Simulation, vol. 14, no. 1, pp. 119-132, 2009.

[32] M. Idrees, S. Islam, S. Haq, and S. Islam, "Application of the optimal homotopy asymptotic method to squeezing flow," Computers \& Mathematics with Applications, vol. 59, no. 12, pp. 3858-3866, 2010.

[33] J. K. Zhou, Deferential Transformation and its Application for Electrical Circuits, Huazhong University press, Wuhan, China, 1986.

[34] S.-U. Islam, S.-U. Haq, and J. Ali, "Numerical solution of special 12th-order boundary value problems using differential transform method," Communications in Nonlinear Science and Numerical Simulation, vol. 14, no. 4, pp. 1132-1138, 2009. 


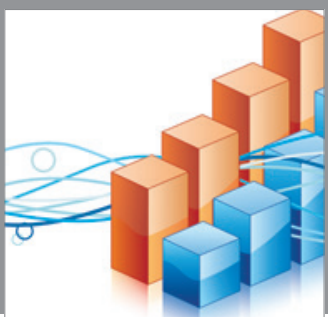

Advances in

Operations Research

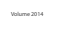

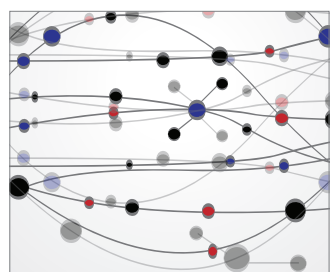

\section{The Scientific} World Journal
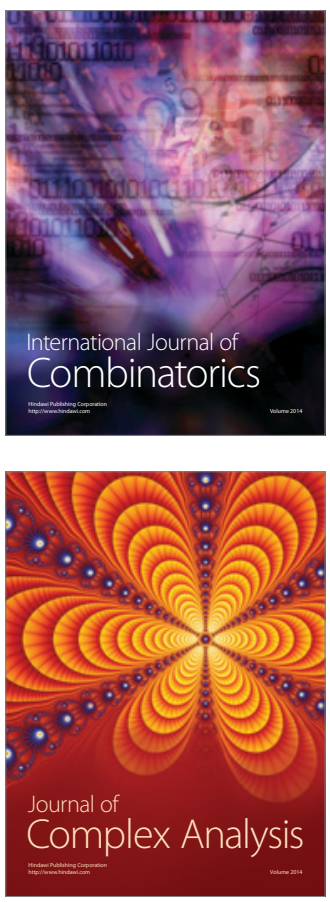

International Journal of

Mathematics and

Mathematical

Sciences
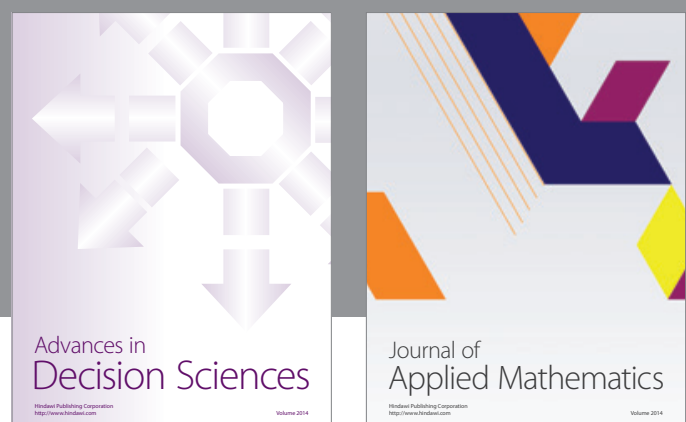

Journal of

Applied Mathematics
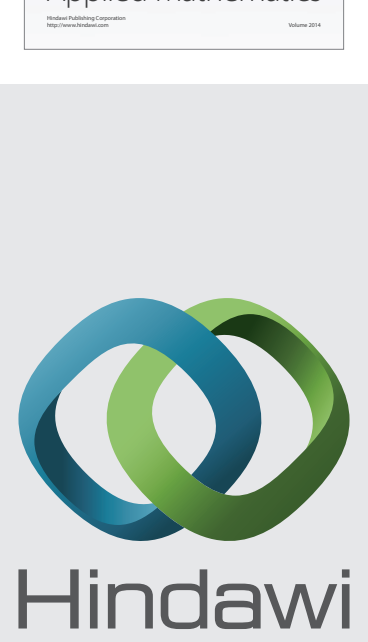

Submit your manuscripts at http://www.hindawi.com
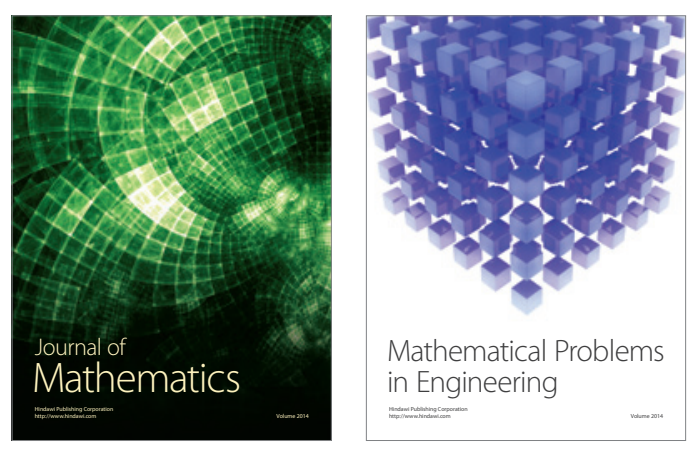

Mathematical Problems in Engineering
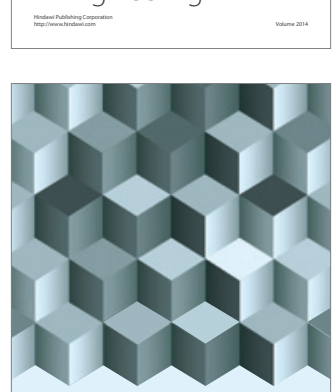

Journal of

Function Spaces
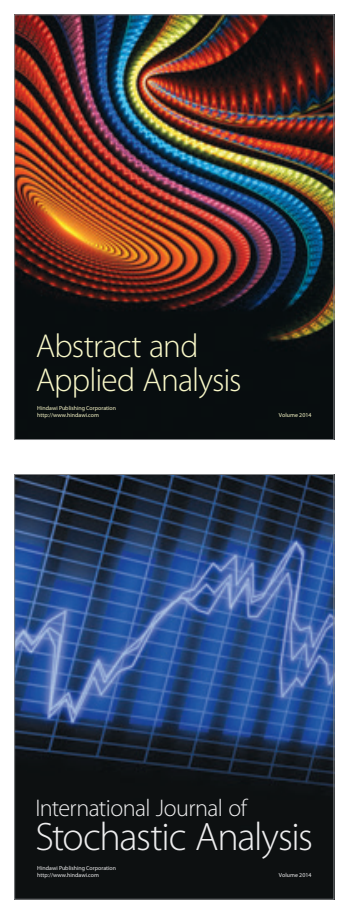

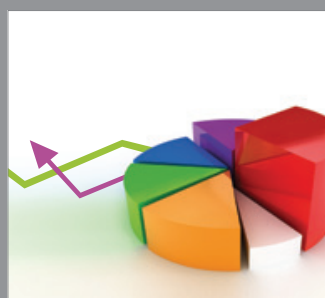

ournal of

Probability and Statistics

Promensencen
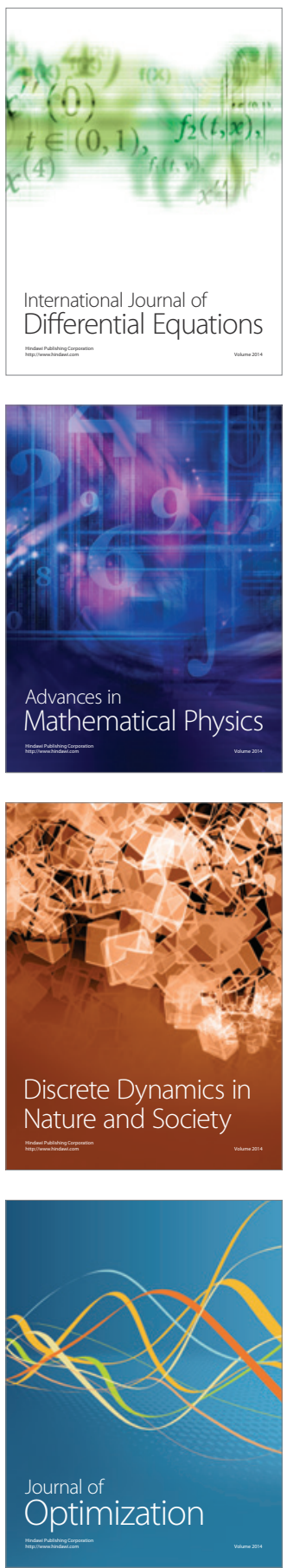